\title{
Information-Theoretic Lower Bounds on the Routing Overhead in Mobile Ad-hoc Networks
}

\author{
Nianjun Zhou \\ Dept. of Electrical, Computer and \\ Systems Engineering \\ 110 Eighth Street \\ Rensselaer Polytechnic Institute \\ Troy, New York 12180, USA \\ e-mail: zhoun@rpi.edu
}

\section{Extended Abstract}

In coding theory, a channel coding algorithm is good if it achieves the Shannon capacity [1]. Similarly, we seek to derive a universal curve against which we can measure how good (or bad) a variable topology routing protocol (e.g. for ad-hoc networks) performs, in comparison with a theoretical minimum routing overhead, which is the amount of information needed to describe the changes in a dynamic network topology.

As a first step in this direction, we consider the following abstract model for the class of hierarchical routing protocols. A network of identical but distinguishable nodes lies within a bounded region of space. Each node moves independently. The whole region is divided into $M$ non-overlapping sub-regions. Each sub-region has its own identifier. There are two levels of hierarchy for nodes. All the nodes of a subregion form a cluster and randomly select a cluster head from the nodes within the same sub-region. It is possible that a sub-region becomes empty and thus will not have a cluster or a cluster head. Each sub-region has a fixed number of neighbors $K$.

A cluster-head maintains two types of topology information: (i) Local cluster topology information, denoted by $I_{L}$, which describes a snapshot of the topology within the boundaries of its sub-region, and (ii) Global topology information, denoted by $I_{G}$, which describes the aggregated topology of the whole network. A cluster-head needs to maintain only a simplified aggregated view of the global topology. This can be described simply as a table that lists, for each sub-region, the member nodes and the cluster-head. Node movements within the same region do not change the cluster membership, and hence do not affect $I_{G}$. For simplicity, we also assume that local node movements do not affect $I_{L}$ i.e. that the local topology is uniquely defined by the identity of the nodes within the subregion and the identity of the cluster-head.

Theorem 1

$$
I_{G}=\log \left(\sum_{k=1}^{\min (N, M)} \frac{N ! M !}{k !(M-k) !(N-k) !} k^{N-k}\right) .
$$

Using the maximum entropy method on (1) yields

$$
N \log M \leq I_{G} \leq N \log M+M \log \left\lceil\frac{N}{M}\right\rceil
$$

which can be interpreted as follows. The lower bound in (2) is the minimum information needed to describe the network without identifying which nodes are the routing nodes. The introduction of routing nodes increases the complexity of the network by $M \log \left\lceil\frac{N}{M}\right\rceil$.

\author{
Alhussein A. Abouzeid \\ Dept. of Electrical, Computer and \\ Systems Engineering \\ 110 Eighth Street \\ Rensselaer Polytechnic Institute \\ Troy, New York 12180, USA \\ e-mail: abouzeid@ecse.rpi.edu
}

\section{Theorem 2:}

$$
I_{L}=\log \left(\left(2^{N-1}\right) N+1\right) .
$$

For large $N, I_{L}=N$ and $\frac{M I_{L}}{I_{G}} \approx \frac{M}{\log M} ; N \gg 1$.

The above results do not take into account the mobility pattern of nodes, and hence all topologies are equally likely. We now consider the following simple Markov mobility model. For a given time-step $\tau$, assume that the probability that a mobile node will stay in the same sub-region is $0 \leq p_{0} \leq 1$, and leave its current sub-region is $1-p_{0}$. We further assume that a node moves to any of the $K$ neighboring sub-regions with equal probability $\frac{1-p_{0}}{K}$ (i.e. a random walk).

Given the mobility pattern of the nodes, it is possible to reduce the uncertainty about the future location of the nodes, and hence the future topologies, given the current topologies, hence reducing the overhead. Let $I_{G}^{P}$ and $I_{L}^{P}$ denote the information overhead with prediction for global and local topology tracking, respectively.

Theorem 3:

$$
N I \leq I_{G}^{P} \leq N I+M \log \left\lceil\frac{N}{M}\right\rceil
$$

where

$$
I=-1\left(p_{0} \log p_{0}+\left(1-p_{0}\right) \log \left(\frac{1-p_{0}}{k}\right)\right) .
$$

The case where $p_{0}=1 /(K+1)$ maximizes $I$, and after some algebraic manipulations, one can show that $I_{G}^{P} / I_{G} \leq \log (K+$ 1) $/ \log M ; N>>1$.

Theorem 4: Assuming each node is equally likely to belong to any of the sub-regions with probability $\frac{1}{M}$,

$I_{L}^{P} \leq-M N\left(\frac{1}{M} \log \frac{1}{M}+\left(1-\frac{1}{M}\right) \log \left(1-\frac{1}{M}\right)\right)+M \log N$

After some algebraic manipulations,

$$
\frac{I_{L}^{P}}{I_{L}} \leq \frac{N \log M+M \log N}{N M} ; M \gg 1 ; N \gg 1
$$

The next step is to optimize the routing protocol based on the frequency of exchange of the above information and the number of hops that the information traverses. These results will be included in the full version of our work for the class of locally proactive/globally reactive protocols.

\section{REFERENCES}

[1] C.E. Shannon. A mathematical theory of communication (2 parts). Bell System Technical Journal, 27:379-423, 623-56, July and October 1948. 